PROCEEDINGS OF THE

AMERICAN MATHEMATICAL SOCIETY

Volume 137, Number 6, June 2009, Pages 2027-2036

S 0002-9939(09)09794-9

Article electronically published on January 16, 2009

\title{
LIPSCHITZNESS OF THE LEMPERT AND GREEN FUNCTIONS
}

\author{
NIKOLAI NIKOLOV, PETER PFLUG, AND PASCAL J. THOMAS \\ (Communicated by Mei-Chi Shaw)
}

\begin{abstract}
Necessary and sufficient conditions for Lipschitzness of the Lempert and Green functions are found in terms of their boundary behaviors.
\end{abstract}

\section{INTRODUCTION AND RESULTS}

By $\mathbb{D}$ we denote the unit disc in $\mathbb{C}$. Let $D$ be a domain in $\mathbb{C}^{n}$. Recall first the definitions of the Lempert function and the Kobayashi-Royden pseudometric of $D$ :

$$
\begin{aligned}
l_{D}(z, w) & :=\inf \{\alpha \in[0,1): \exists \varphi \in \mathcal{O}(\mathbb{D}, D): \varphi(0)=z, \varphi(\alpha)=w\}, \\
\kappa_{D}(z ; X) & :=\inf \left\{\alpha \geq 0: \exists \varphi \in \mathcal{O}(\mathbb{D}, D): \varphi(0)=z, \alpha \varphi^{\prime}(0)=X\right\} .
\end{aligned}
$$

We point out that both functions are upper semicontinuous and $l_{D}$ is symmetric. The Kobayashi-Buseman pseudometric $\hat{\kappa}_{D}(z ; \cdot)$ (the Kobayashi pseudodistance $\left.k_{D}\right)$ is the largest pseudonorm (pseudodistance) which does not exceed $\kappa_{D}(z ; \cdot)$ $\left(\tanh ^{-1} l_{D}\right)$. Note that if $D$ is a taut domain, i.e., $\mathcal{O}(\mathbb{D}, D)$ is a normal family, then $\kappa_{D}$ and $\hat{\kappa}_{D}$ are the infinitesimal forms of $l_{D}$ and $k_{D}$, respectively (see 9 , Theorem 1 for a more general result). Moreover, recall (cf. [7, Proposition 3.2) that $D$ is a taut domain if and only if

$$
\lim _{z \in K, w \rightarrow \partial D} l_{D}(z, w)=1 \text { for any } K \Subset D .
$$

(Note that for an unbounded $D$ the point $\infty$ belongs, by definition, to $\partial D$.)

The main result in [6] (see Theorem 6 there) is that $\kappa_{D}$ is locally a Hölder function of order $2 / 3$ on any $C^{6}$-smooth strongly pseudoconvex domain $D$ in $\mathbb{C}^{n}$ (see also [5], where it is claimed that $\kappa_{D}$ is locally Lipschitz but the proof there seems to be not correct).

Our first goal in the present note is to generalize this result showing that $l_{D}$ and $\kappa_{D}$ are Lipschitz functions under a natural assumption about the boundary behavior of $l_{D}$. In fact we have the following result 11

Received by the editors June 9, 2008.

2000 Mathematics Subject Classification. Primary 32F45, 32U35.

Key words and phrases. Lipschitzness, Lempert function, Kobayashi-Royden pseudometric, pluricomplex Green function, Azukawa pseudometric.

This paper was started during the stay of the first-named author at the Carl von Ossietzky Universität, Oldenburg (October 2007; supported by a grant from the DFG, Az. PF 227/9-1), and was finished during his stay at the Université Paul Sabatier, Toulouse (January 2008).

${ }^{1}$ Proofs for this and the next results will be presented in section 2 .

(C)2009 American Mathematical Society Reverts to public domain 28 years from publication 
Proposition 1. Let $D \subset \mathbb{C}^{n}$ be a hyperbolic domain (i.e., $k_{D}$ is a distance) and $K \Subset D$ be such that $\sup _{z \in K, w \in D} \frac{1-l_{D}(z, w)}{\operatorname{dist}(w, \partial D)}<\infty$. Then:

(i) $l_{D}$ is a Lipschitz function on $K \times D$;

(ii) there is a $C>0$ such that if $z, w \in K, X, Y \in \mathbb{C}^{n}$, then

$$
\left|\kappa_{D}(z ; X)-\kappa_{D}(w ; Y)\right| \leq C((\|X\|+\|Y\|) \cdot\|z-w\|+\|X-Y\|) .
$$

Remark A. (a) By symmetry, $l_{D}$ is a Lipschitz function on $D \times K$, too. On the other hand, $l_{\mathbb{D}}$ is not a Lipschitz function on $\mathbb{D} \times \mathbb{D}$.

(b) For the Carathéodory-Reiffen pseudometric, the same estimate as in (ii) remains true for any domain in $\mathbb{C}^{n}$ (see [3], Proposition 2.5.1(c)).

(c) Any compact subset of a strongly pseudoconvex domain satisfies the assumption of Proposition [1 (cf. 3], Theorem 10.2.1).

(d) If $D \subset \mathbb{C}^{n}$ is a hyperbolic domain, $K \Subset D, L \Subset \mathbb{C}^{n}$, and $\sup _{z \in K, w \in D} \frac{1-l_{D}(z, w)}{\operatorname{dist}^{\alpha}(w, \partial D)}$ $<\infty, \alpha \in(0,1)$, then obvious modifications in the proof of Proposition 11imply that $l_{D}$ and $\kappa_{D}$ are Hölder functions with exponent $\alpha$ on $K \times D$ and $K \times L$, respectively. On the other hand, $\alpha$ cannot be taken larger than 1 ; one can show that for any domain $D \subsetneq \mathbb{C}^{n}$ and any point $z \in D$ we have $\limsup _{w \rightarrow \partial D} \frac{1-l_{D}(z, w)}{\operatorname{dist}(w, \partial D)}>0$.

We point out that for a taut domain $D$ the assumption of Proposition 1 is also necessary for $l_{D}$ to be a Lipschitz function.

Corollary 2. Let $D \subset \mathbb{C}^{n}$ be a taut domain and $K \Subset D$. Then $\sup _{z \in K, w \in D} \frac{1-l_{D}(z, w)}{\operatorname{dist}(w, \partial D)}$ $<\infty$ if and only if $l_{D}$ is a Lipschitz function on $K \times D$.

To prove the Lipschitzness of $\hat{\kappa}_{D}$ under the assumption of Proposition 1, we shall need the following result.

Proposition 3. Let $D \subset \mathbb{C}^{n}$ be a hyperbolic domain and let $K \Subset D, c>0$ be such that

$$
\left|\kappa_{D}(z ; X)-\kappa_{D}(w ; X)\right| \leq c\|X\| \cdot\|z-w\|, \quad z, w \in K, X \in \mathbb{C}^{n} .
$$

Then there is a $C>0$ such that if $z, w \in K, X, Y \in \mathbb{C}^{n}$, then

$$
\left|\hat{\kappa}_{D}(z ; X)-\hat{\kappa}_{D}(w ; Y)\right| \leq C((\|X\|+\|Y\|) \cdot\|z-w\|+\|X-Y\|) .
$$

The next corollary is an immediate consequence of Propositions 1 and 3 ,

Corollary 4. Let $D \subset \mathbb{C}^{n}$ and $K \Subset D$ be as in Proposition 1. Then there is a $C>0$ such that if $z, w \in K, X, Y \in \mathbb{C}^{n}$, then

$$
\left|\hat{\kappa}_{D}(z ; X)-\hat{\kappa}_{D}(w ; Y)\right| \leq C((\|X\|+\|Y\|) \cdot\|z-w\|+\|X-Y\|) .
$$

The second aim of our paper is to find a necessary and sufficient condition for the exponential of the pluricomplex Green function to be Lipschitz (similar to that for the Lempert function).

Recall first the definitions of the pluricomplex Green function and the Azukawa pseudometric of a domain $D$ in $\mathbb{C}^{n}$ :

$$
\begin{gathered}
g_{D}(z, w):=\sup \left\{u(w): u \in P S H(D), u<0, \limsup _{\zeta \rightarrow z}(u(\zeta)-\log \|\zeta-z\|)<\infty\right\}, \\
A_{D}(z ; X):=\limsup _{t \rightarrow 0} \frac{\tilde{g}_{D}(z, z+t X)}{|t|},
\end{gathered}
$$


where $\tilde{g}_{D}:=\exp g_{D}$. We point out that both functions are upper semicontinuous (cf. 4, page 10) and $\tilde{g}_{D} \leq l_{D}$. Note also that, in general, $g_{D}$ is not symmetric.

Recall also that a domain $D \subset \mathbb{C}^{n}$ is called hyperconvex if it has a negative plurisubharmonic exhaustion function. The next proposition is a consequence of the proof of Theorem 3.1 in 2] (see also [1, Theorem 2, for a weaker version).

Proposition 5. Let $D \subset \mathbb{C}^{n}$ be a bounded domain. Then the following conditions are equivalent:

(i) there is $u \in P S H(D)$ with $u<0$ and $\inf _{z \in D} u(z) / \operatorname{dist}(z, \partial D)>-\infty$;

(ii) $D$ is hyperconvex and there are $z_{0} \in D$ and $C>0$ such that if $w_{1}, w_{2} \in$ $D \backslash\left\{z_{0}\right\}$, then

$$
\left|g_{D}\left(z_{0}, w_{1}\right)-g_{D}\left(z_{0}, w_{2}\right)\right| \leq C \frac{\left\|w_{1}-w_{2}\right\|}{\min \left\{\left\|z_{0}-w_{1}\right\|,\left\|z_{0}-w_{2}\right\|\right\}} ;
$$

(iii) $D$ is hyperconvex and for any $K \Subset D$ there is a $C>0$ such that if $z \in K$ and $w_{1}, w_{2} \in D \backslash\{z\}$, then

$$
\left|g_{D}\left(z, w_{1}\right)-g_{D}\left(z, w_{2}\right)\right| \leq C \frac{\left\|w_{1}-w_{2}\right\|}{\min \left\{\left\|z-w_{1}\right\|,\left\|z-w_{2}\right\|\right\}} .
$$

As a simple consequence we get the following result for $\tilde{g}_{D}$.

Corollary 6. Let $D$ and $u$ be as in Proposition $5(i)$ and let $K \Subset D$. Then there is $C>0$ such that

$$
\left|\tilde{g}_{D}\left(z, w_{1}\right)-\tilde{g}_{D}\left(z, w_{2}\right)\right| \leq C\left\|w_{1}-w_{2}\right\|, \quad z \in K, w_{1}, w_{2} \in D .
$$

Remark B. Let $D$ be a hyperconvex domain (not necessary bounded) and $u$ be as in Proposition 5 (if $D$ is bounded, then (i) implies that $u$ is an exhaustion function of $D$ and hence $D$ is hyperconvex). Then, for an arbitrary $K \Subset D$, the assumptions of Proposition 1 are satisfied. Indeed, it follows from (5) below that

$$
\begin{aligned}
1-l_{D}(z, w) \leq 1-\tilde{g}_{D}(z, w) \leq-g_{D}(z, w) \leq c \operatorname{dist}(w, \partial D), & \\
& z \in K, w \in D, \text { near } \partial D ;
\end{aligned}
$$

hence the inequality in the assumption of Proposition 1 is fulfilled. It remains to use that $l_{D} \geq \tilde{g}_{D}$ and that $D$ is hyperconvex. Hence $D$ is taut (cf. [7, page 607) and therefore hyperbolic.

From Corollary 6 we get that, under the same assumptions, $\tilde{g}_{D}$ and $A_{D}$ are Lipschitz functions (in both arguments).

Proposition 7. Let $D$ and $u$ be as in Proposition $5($ i) and let $K \subset D$ be compact. Then:

(i) $\tilde{g}_{D}$ is a Lipschitz function on $K \times D$;

(ii) there is a $C>0$ such that if $z, w \in K, X, Y \in \mathbb{C}^{n}$, then

$$
\left|A_{D}(z ; X)-A_{D}(w ; Y)\right| \leq C((\|X\|+\|Y\|) \cdot\|z-w\|+\|X-Y\|) .
$$

It remains an open question whether $\tilde{g}_{D}$ is a Lipschitz function on $D \times K$.

Remark C. Let $D \subset \mathbb{C}^{n}$ be a pseudoconvex balanced domain with Minkowski function $h_{D}$. Recall that (cf. [3, Propositions 3.1.10 and 4.2.7 (b))

$$
l_{D}(0, \cdot)=\kappa_{D}(0 ; X)=g_{D}(0, \cdot)=A_{D}(0 ; \cdot)=h_{D} .
$$

Note also that (cf. 7], Proposition 4.4) $D$ is taut $\Leftrightarrow D$ is hyperconvex $\Leftrightarrow D$ is bounded and $h_{D}$ is continuous. 
By Corollary 2 or Corollary 6, for a taut balanced domain $D$ the following are equivalent:

(i) there is $c>0$ such that $1-h_{D}(z) \leq c \cdot \operatorname{dist}(z, \partial D), z \in D$;

(ii) there is $c^{\prime}>0$ such that $\left|h_{D}(z)-h_{D}(w)\right| \leq c^{\prime}\|z-w\|, z, w \in D$.

(Taking $h_{D}(z)=\left|z_{1}\right|+\left|z_{2}\right|+\sqrt{\left|z_{1} z_{2}\right|}$ provides an example of a taut balanced domain $D \subset \mathbb{C}^{2}$ which does not have the above properties.)

We point out that $(i) \Leftrightarrow(i i)$ with $c=c^{\prime}$ for any balanced domain $D$ in $\mathbb{C}^{n}$.

Indeed, assume that $(i)$ holds. Then for any $z, w$ with $1>h_{D}(z)>h_{D}(w)$ we have

$$
\begin{aligned}
& h_{D}(z)-h_{D}(w)=h_{D}(z)\left(1-h_{D}\left(w / h_{D}(z)\right)\right) \leq h_{D}(z) c \cdot \operatorname{dist}\left(w / h_{D}(z), \partial D\right) \\
& \quad \leq h_{D}(z) c\left\|w / h_{D}(z)-z / h_{D}(z)\right\|=c\|z-w\| .
\end{aligned}
$$

Conversely, assume that (ii) is true. Fix a $z \in D$. If $\|u\|<r_{z}:=\left(1-h_{D}(z)\right) / c^{\prime}$, then $h_{D}(z+u) \leq h_{D}(z)+c^{\prime}\|u\|<1$, which shows that $\mathbb{B}_{n}\left(z, r_{z}\right) \subset D$. Hence $\operatorname{dist}(z, \partial D) \geq r_{z}$; that is, (i) holds with $c=c^{\prime}$.

\section{Proofs}

Proof of Proposition 1. The assumption of Proposition 1 means that there is a $c>0$ such that for any $r \in(0,1)$ and $\varphi \in \mathcal{O}(\mathbb{D}, D)$ with $\varphi(0) \in K$ one has that

$$
c \cdot \operatorname{dist}(\varphi(r \mathbb{D}), \partial D) \geq 1-r .
$$

Note that there is a $c_{1}>0$ such that

$$
c_{1}\|z-w\| \geq l_{D}(z, w), \quad z \in K, w \in D .
$$

On the other hand, if $D$ is unbounded, then, by hyperbolicity,

$$
m^{*}=\liminf _{z \in K, w \rightarrow \infty} l_{D}(z, w)>0
$$

(use e.g. 7], Proposition 3.1). Fix an $m \in\left(0, \min \left\{1 / 2, m^{*}\right\}\right)$. Then, again by hyperbolicity, we find a $c_{2}>0$ such that

$$
l_{D}(z, w) \leq m, z \in K, w \in D \Rightarrow l_{D}(z, w) \geq c_{2}\|z-w\|
$$

(apply e.g. [3, Theorem 7.2 .2 ; if $D$ is bounded, the last inequality holds even on $K \times D$ with suitable $c_{2}>0$; no other assumptions are needed in this situation). We may assume that $c_{1}>1>c_{2}$. Set $c_{3}=c_{1}\left(1+c /\left(m c_{2}\right)\right)$. To prove (i), it suffices to show that if

$$
\left|l_{D}\left(z, w_{1}\right)-l_{D}\left(z, w_{2}\right)\right| \leq c_{3}\left\|w_{1}-w_{2}\right\|, z \in K, w_{1}, w_{2} \in D,
$$

then

$$
\left|l_{D}\left(w_{1}, z\right)-l_{D}\left(w_{2}, z\right)\right| \leq 2 c_{3}\left\|w_{1}-w_{2}\right\|, w_{1}, w_{2} \in K, z \in D .
$$

To prove (11), we may assume that $\alpha:=l_{D}\left(z, w_{1}\right) \leq l_{D}\left(z, w_{2}\right)$ and $z \neq w_{1}$. Then, by hyperbolicity, $\alpha>0$. Set $r=1-c\left\|w_{1}-w_{2}\right\| / \alpha$. We shall consider three cases.

Case 1. $r>\max \{\alpha, m\}$. Then for any $\alpha^{\prime} \in(\alpha, r)$ there is $\varphi \in \mathcal{O}(\mathbb{D}, D)$ with $\varphi(0)=z$ and $\varphi\left(\alpha^{\prime}\right)=w_{1}$. Set $\psi(\zeta)=\varphi(r \zeta)+\left(w_{2}-w_{1}\right) r \zeta / \alpha^{\prime}, \zeta \in \mathbb{D}$. Then $\psi \in \mathcal{O}(\mathbb{D}, D)$ and $\psi\left(\alpha^{\prime} / r\right)=w_{2}\left(\alpha^{\prime}<r\right)$. It follows that $l_{D}\left(z, w_{2}\right) \leq \alpha / r$ and hence

$$
\begin{aligned}
& l_{D}\left(z, w_{2}\right)-l_{D}\left(z, w_{1}\right) \leq \alpha(1-r) / r \\
& \quad=c\left\|w_{2}-w_{1}\right\| / r \leq c\left\|w_{2}-w_{1}\right\| / m \leq c_{3}\left\|w_{2}-w_{1}\right\| .
\end{aligned}
$$


Case 2. $\alpha \geq \max \{r, m\}$. Then

$$
\begin{aligned}
& l_{D}\left(z, w_{2}\right)-l_{D}\left(z, w_{1}\right)<1-\alpha \leq 1-r \\
& \quad=c\left\|w_{1}-w_{2}\right\| / \alpha \leq c\left\|w_{1}-w_{2}\right\| / m<c_{3}\left\|w_{1}-w_{2}\right\| .
\end{aligned}
$$

Case 3. $m \geq \max \{r, \alpha\}$. Then

$$
\left\|w_{1}-w_{2}\right\|=(1-r) \alpha / c \geq(1-m) \alpha / c \geq(1-m) c_{2}\left\|z-w_{1}\right\| / c,
$$

and, by the triangle inequality, $\left\|z-w_{2}\right\| \leq\left(1+c /\left((1-m) c_{2}\right)\right)\left\|w_{1}-w_{2}\right\|$. Since $m \leq 1 / 2$, it follows that

$$
l_{D}\left(z, w_{2}\right)-l_{D}\left(z, w_{1}\right)<l_{D}\left(z, w_{2}\right) \leq c_{1}\left\|z-w_{2}\right\| \leq c_{3}\left\|w_{1}-w_{2}\right\| .
$$

This completes the proof of (1).

The proof of (2) is similar to that of (10) and we sketch it. We may assume that $0<\beta:=l_{D}\left(w_{1}, z\right) \leq l_{D}\left(w_{2}, z\right)$ and then set $s=1-2 c\left\|w_{1}-w_{2}\right\| / \beta$. We get as above that:

Case 1. If $\beta \geq \max \{s, m\}$, then $l_{D}\left(w_{2}, z\right)-l_{D}\left(w_{1}, z\right)<2 c\left\|w_{1}-w_{2}\right\| / \beta$;

Case 2. If $m \geq \max \{s, \beta\}$, then $l_{D}\left(w_{2}, z\right)-l_{D}\left(w_{1}, z\right)<c_{3}\left\|w_{1}-w_{2}\right\|$.

Case 3. In the remaining case $s>\max \{\beta, m\}$, for any $\beta^{\prime} \in(\beta, s)$ we may find $\varphi \in \mathcal{O}(\mathbb{D}, D)$ with $\varphi(0)=w_{1}$ and $\varphi\left(\beta^{\prime}\right)=z_{1}$. Set $\psi(\zeta)=\varphi(s \zeta)+\left(w_{2}-w_{1}\right)(1-$ $\left.s \zeta / \beta^{\prime}\right), \zeta \in \mathbb{D}$. Then $\psi \in \mathcal{O}(\mathbb{D}, D), \psi(0)=w_{2}$ and $\psi\left(\beta^{\prime} / s\right)=z$. It follows that $l_{D}\left(w_{2}, z\right) \leq \beta / s$ and hence

$$
l_{D}\left(w_{2}, z\right)-l_{D}\left(w_{1}, z\right) \leq 2 c\left\|w_{2}-w_{1}\right\|,
$$

which completes the proof of (2).

Next, we shall prove (ii). It is enough to show that

$$
\left|\kappa_{D}(z ; X)-\kappa_{D}(w ; X)\right| \leq 4 c c_{4}\|X\| \cdot\|z-w\|
$$

and

$$
\left|\kappa_{D}(z ; X)-\kappa_{D}(z ; Y)\right| \leq c_{5}\|X-Y\|,
$$

for any $z, w \in K, X, Y \in \mathbb{C}^{n}$, where $c_{4}:=\sup _{u \in K,\|U\|=1} \kappa_{D}(u ; U), c_{5}:=c_{4}(1+$ $\left.2 c / c_{6}\right)$ and $c_{6}:=\inf _{u \in K,\|U\|=1} \kappa_{D}(u ; U)\left(c_{6}>0\right.$ by hyperbolicity; cf. [3], Theorem 7.2.2).

For proving (3), observe that

$$
\left|\kappa_{D}(z ; X)-\kappa_{D}(w ; X)\right| \leq 2 c_{4}\|X\| .
$$

So (3) is trivial if $p=1-c\|z-w\| \leq 1 / 2$. Otherwise, we may assume that $\kappa_{D}(z ; X) \leq \kappa_{D}(w ; X)$. For any $\varphi \in(\mathbb{D}, D)$ set $\psi(\zeta)=\varphi(p \zeta)+w-z, \zeta \in \mathbb{D}$. Then $\psi \in \mathcal{O}(\mathbb{D}, D)$, which shows that $\kappa_{D}(w ; X) \leq \kappa_{D}(z ; X) / p$. This implies (3) with $2 c c_{4}$ instead of $4 c c_{4}$.

To get (4), we may assume that $\gamma=\kappa_{D}(z ; X) \leq \kappa_{D}(z ; Y)$ and $X \neq 0$. Then $\gamma>0$. For $q=1-c\|X-Y\| / \gamma$ we have two cases.

Case 1. $q>1 / 2$. Let $\varphi \in \mathcal{O}(\mathbb{D}, D)$ be such that $\varphi(0)=z$ and $\gamma^{\prime} \varphi^{\prime}(0)=X$ for some $\gamma^{\prime}$. Set $\psi(\zeta)=\varphi(q \zeta)+(Y-X) q \zeta / \gamma^{\prime}$. Then $\psi \in \mathcal{O}(\mathbb{D}, \mathbb{D})$ and $\gamma^{\prime} \psi^{\prime}(0)=q Y$. It follows that $\kappa_{D}(z ; Y) \leq \gamma / q$ and hence

$$
\kappa_{D}(z ; Y)-\kappa_{D}(z ; X) \leq \gamma(1-q) / q=c\|X-Y\| / q \leq c_{5}\|X-Y\| .
$$


Case 2. $q \leq 1 / 2$. Then $\|X-Y\|=(1-q) \gamma / c \geq c_{6}\|X\| /(2 c)$ and, by the triangle inequality, $\|Y\| \leq\left(1+2 c / c_{6}\right)\|X-Y\|$. It follows that

$$
\kappa_{D}(z ; Y)-\kappa_{D}(z ; X)<\kappa_{D}(z ; Y) \leq c_{4}\|Y\| \leq c_{5}\|X-Y\| .
$$

This completes the proof of Proposition 1 .

Proof of Corollary 2, By Proposition 1, it is enough to show that if

$$
\left|l_{D}\left(z, w_{1}\right)-l_{D}\left(z, w_{2}\right)\right| \leq c\left\|w_{1}-w_{2}\right\|, \quad z \in K, w_{1}, w_{2} \in D,
$$

then

$$
\sup _{z \in K, w \in D} \frac{1-l_{D}(z, w)}{\operatorname{dist}(w, \partial D)}<\infty
$$

Suppose this is not true. Then there are sequences $\left(z_{j}\right)_{j} \subset K$ and $\left(w_{j}\right)_{j} \subset D$ such that

$$
1-l_{D}\left(z_{j}, w_{j}\right) \geq j \operatorname{dist}\left(w_{j}, \partial D\right), \quad j \in \mathbb{N} .
$$

Choose $b_{j} \in \partial D$ with $\left\|w_{j}-b_{j}\right\|=\operatorname{dist}\left(w_{j}, \partial D\right)$ and sequences $\left(b_{j, k}\right)_{k} \subset D$ with $b_{j, k} \rightarrow b_{j}$ if $k \rightarrow \infty, j \in \mathbb{N}$. Then

$$
\begin{aligned}
j \leq \frac{1-l_{D}\left(z_{j}, b_{j, k}\right)+l_{D}\left(z_{j}, b_{j, k}\right)-}{\| w_{D}\left(z_{j}, w_{j}\right)} & \\
\qquad w_{j}-b_{j} \| & \leq \frac{1-l_{D}\left(z_{j}, b_{j, k}\right)}{\left\|w_{j}-b_{j}\right\|}+c \frac{\left\|w_{j}-b_{j, k}\right\|}{\left\|w_{j}-b_{j}\right\|} \leq 1+2 c
\end{aligned}
$$

if $k=k_{j}$ is sufficiently large. Recall that $D$ is taut; therefore such a $k_{j}$ always exists, a contradiction.

Proof of Proposition 3. Since $\hat{\kappa}_{D}(z ; \cdot)$ is a norm, it is enough to show that for any $K \Subset D$ there is $C^{\prime}>0$ such that

$$
\left|\hat{\kappa}_{D}(z ; X)-\hat{\kappa}_{D}(w ; X)\right| \leq C^{\prime}\|X\| \cdot\|z-w\|, \quad z, w \in K, X \in \mathbb{C}^{n} .
$$

We may assume that $z \neq w, X \neq 0$ and $\hat{\kappa}_{D}(z ; X) \leq \hat{\kappa}_{D}(w ; X)$. Then there are vectors $X_{1}, \ldots, X_{2 n-1} \in \mathbb{C}^{n}$ with sum $X$ such that (see [8], Theorem 1)

$$
\sum_{j=1}^{2 n-1} \kappa_{D}\left(z ; X_{j}\right) \leq \hat{\kappa}_{D}(z ; X)+\|X\| \cdot\|z-w\| .
$$

It follows that

$$
\begin{aligned}
0 & \leq \hat{\kappa}_{D}(w ; X)-\hat{\kappa}_{D}(z ; X) \leq \sum_{j=1}^{2 n-1}\left(\kappa_{D}\left(w ; X_{j}\right)-\kappa_{D}\left(z ; X_{j}\right)\right)+\|X\| \cdot\|z-w\| \\
& \leq\|z-w\|\left(\|X\|+c \sum_{j=1}^{2 n-1}\left\|X_{j}\right\|\right) .
\end{aligned}
$$

It remains to use that $\sum_{j=1}^{2 n-1}\left\|X_{j}\right\| \leq \frac{c_{4}}{c_{6}}\|X\|$, where $c_{4}$ and $c_{6}$ are as in the proof of Proposition 1 .

Proof of Proposition 5. (ii) $\Rightarrow$ (i). Put $u=\tilde{g}_{D}\left(z_{0}, \cdot\right)-1$. Since $D$ is a hyperconvex domain, then $\lim _{w \rightarrow \partial D} u(w)=0$. Now a similar argument as in the proof of Corollary 2 implies that $u$ has the required property.

Since (iii) $\Rightarrow$ (ii) is trivial, it remains to prove: 
(i) $\Rightarrow$ (iii). Since $u$ is an exhaustion function of $D$, it follows that $D$ is hyperconvex.

Fix a $K \Subset D$. We shall show that if $D$ is hyperconvex (not necessarily bounded) and $u$ is as in (i), then

$$
\liminf _{z \in K, w \rightarrow \partial D} g_{D}(z, w) / \operatorname{dist}(z, \partial D)>-\infty .
$$

Indeed, let $\tilde{u}$ be an exhaustion function of $D$ and $\hat{u}=\max \{u, \tilde{u}\}$. Then take a domain $G_{1} \Subset D, K \Subset G_{1}$, and put $\varepsilon=\sup _{G_{1}} \hat{u} / 2<0$. Next we choose a domain $G_{2} \Subset D, G_{1} \Subset G_{2}$, such that $\inf _{\partial G_{2}} \hat{u} \geq \varepsilon$.

Fix a $z \in K$. Set $\varphi(z, \cdot)=\log \left(\|\cdot-z\| / \operatorname{diam} G_{2}\right), m=\inf _{K \times G_{1}} \varphi$ and

$$
v_{z}= \begin{cases}\varphi(z, \cdot)+m & \text { on } G_{1} \\ \max \{\varphi(z, \cdot)+m, m \hat{u} / \varepsilon\} & \text { on } G_{2} \backslash G_{1} \\ m \hat{u} / \varepsilon & \text { on } D \backslash G_{2} .\end{cases}
$$

It is easy to check that $v_{z} \in P S H(D)$ for $z \in K$. Hence $g_{D}(z, \cdot) \geq v_{z}$, which implies (5).

Now let $r>0$ be such that $\mathbb{B}(a, r) \subset D$ for any $z \in K$. For any $\varepsilon \in(0, r)$ we set

$$
g_{D}^{\varepsilon}(z, w)=\sup \left\{u(w): u \in P S H(D), u<0,\left.u\right|_{\mathbb{B}(z, \varepsilon)} \leq \log (\varepsilon / r)\right\} .
$$

One can easily check that $g_{D}^{\varepsilon}(z, \cdot)$ is a maximal plurisubharmonic function on $D \backslash$ $\overline{\mathbb{B}(z, \varepsilon)}$ (cf. [3], page 383 for this notion),

$$
\max \left\{\log (\varepsilon / r), g_{D}(z, w)\right\} \leq g_{D}^{\varepsilon}(z, w) \leq \log \frac{\max \{\|z-w\|, \varepsilon\}}{r}
$$

and $g_{D}^{\varepsilon}(z, \cdot) \downarrow g_{D}(z, \cdot)$ as $\varepsilon \downarrow 0$ locally uniformly in $D \backslash\{z\}$ (cf. [2], page 338, and Proposition 2.2). Moreover, since $D$ is hyperconvex, $g_{D}^{\varepsilon}$ can be extended as a continuous function on $D \times \bar{D}$ by setting $g_{D \mid D \times \partial D}^{\varepsilon}=0$.

We shall find $c_{1}, c_{2}>0$ such that if $z \in K, w_{1}, w_{2} \in D \backslash\{z\}$, and $\varepsilon>0$ satisfy the inequality

$$
\max \left\{\varepsilon, c_{1}\left\|w_{1}-w_{2}\right\|\right\}<\min \left\{r / 2,\left\|z-w_{1}\right\|,\left\|z-w_{2}\right\|\right\},
$$

then

$$
\left|g_{D}^{\varepsilon}\left(z, w_{1}\right)-g_{D}^{\varepsilon}\left(z, w_{2}\right)\right| \leq c_{2} \frac{\left\|w_{1}-w_{2}\right\|}{\min \left\{\left\|z-w_{1}\right\|,\left\|z-w_{2}\right\|\right\}} .
$$

Assuming (8), take arbitrary points $w_{1}, w_{2} \in D \backslash\{z\}$. To prove (iii), we may assume that $g_{D}^{\varepsilon}\left(z, w_{1}\right) \leq g_{D}^{\varepsilon}\left(z, w_{2}\right)$, where $\varepsilon$ is as above. There is a semicircle with diameter $\left[w_{1} w_{2}\right]$, say $\gamma:[0, \pi] \rightarrow \mathbb{C}^{n}, \gamma(0)=w_{1}, \gamma(\pi)=w_{2}$, such that $\operatorname{dist}(z, \gamma)=$ $\min \left\{\left\|z-w_{1}\right\|,\left\|z-w_{2}\right\|\right\}$. Let $t^{\prime} \in(0,1]$ be the largest number such that $\gamma(t) \in D$ for $t \in\left(0, t^{\prime}\right)$. If $t^{\prime}=1$, then an "integration along $\gamma^{\text {" gives }}$

$$
g_{D}^{\varepsilon}\left(z, w_{2}\right)-g_{D}^{\varepsilon}\left(z, w_{1}\right) \leq \pi c_{2} \frac{\left\|w_{1}-w_{2}\right\|}{\min \left\{\left\|z-w_{1}\right\|,\left\|z-w_{2}\right\|\right\}} .
$$

If $t^{\prime}<1$, then $\gamma\left(t^{\prime}\right) \in \partial D$. Since

$$
\lim _{w \rightarrow \partial D} g_{D}^{\varepsilon}(z, w)=0>g_{D}^{\varepsilon}\left(z, w_{2}\right)>g_{D}^{\varepsilon}\left(z, w_{1}\right)
$$

and $g_{D}^{\varepsilon}$ is continuous, we may find a $t^{*} \in\left[0, t^{\prime}\right)$ with $g_{D}^{\varepsilon}\left(z, \gamma\left(t^{*}\right)\right)=g_{D}^{\varepsilon}\left(z, w_{2}\right)$. Then, similarly as above, we get the same estimates. Letting $\varepsilon \rightarrow 0$ gives the estimate in (iii) with $C=\pi c_{2}$. 
To prove (8), we may assume that $g_{D}^{\varepsilon}\left(z, w_{1}\right)<g_{D}^{\varepsilon}\left(z, w_{2}\right)$. Now let $f=f_{z, w} \in$ $\mathcal{O}(D, \mathbb{D})$ be an extremal function for the Carathéodory distance $c_{D}(z, w)$ (cf. [3], page 16). We may assume that $f(z)=0$. For $z \neq w$ set $h_{z, w}(\zeta)=f(\zeta) / f(w)$, $\zeta \in D$. Then there are $c_{1}, c_{3}>0$ with

$$
\left|h_{z, w}(\zeta)\right| \leq \frac{\tanh c_{D}(z, \zeta)}{\tanh c_{D}(z, w)} \leq c_{1} \frac{\|\zeta-z\|}{\|w-z\|} \leq \frac{c_{3}}{\|w-z\|}, z \in K, w \in D \backslash\{z\}
$$

(use that $\mathbb{B}(z, r) \subset D \subset \mathbb{B}(z, R), z \in K$, for certain $r, R$ ). Set

$$
D^{\prime}=\left\{\zeta \in D: \zeta+h_{z, w_{1}}(\zeta)\left(w_{2}-w_{1}\right) \in D\right\}, \quad D^{\prime \prime}=D^{\prime} \backslash \overline{\mathbb{B}(z, \varepsilon)}
$$

and

$$
\hat{g}(\zeta)=g_{D}^{\varepsilon}\left(z, \zeta+\left(w_{2}-w_{1}\right) h_{z, w_{1}}(\zeta)\right), \quad \zeta \in D^{\prime} .
$$

It follows by (7) and (9) that $\mathbb{B}(z, \varepsilon) \Subset \mathbb{B}(z, r / 2) \Subset D^{\prime}$ and $w_{1} \in D^{\prime \prime}$. On the other hand, by (5), there is a $c_{4}>0$ such that

$$
g_{D}(z, \zeta) \geq-c_{4} \operatorname{dist}(\zeta, \partial D), \quad \zeta \in D \backslash \mathbb{B}(z, r / 2) .
$$

Thus, (6) and (9) imply that

$$
\min _{\zeta \in \partial D^{\prime}} g_{D}^{\varepsilon}(z, \zeta) \geq \min _{\zeta \in \partial D^{\prime}} g_{D}(z, \zeta) \geq-c_{4} \max _{\zeta \in \partial D^{\prime}} \operatorname{dist}(\zeta, \partial D) \geq-c_{3} c_{4} \frac{\left\|w_{2}-w_{1}\right\|}{\left\|w_{1}-z\right\|} .
$$

Then for

we have that

$$
v(\zeta)=\hat{g}(\zeta)-g_{D}^{\varepsilon}(z, \zeta)
$$

$$
\limsup _{\zeta \rightarrow \partial D^{\prime}} v(\zeta) \leq c_{3} c_{4} \frac{\left\|w_{2}-w_{1}\right\|}{\left\|w_{1}-z\right\|} .
$$

On the other hand, for $\zeta \in \partial \mathbb{B}(z, \varepsilon)$, it follows by (6) and (9) that

$$
\begin{aligned}
v(\zeta) & \leq \log \frac{\max \left\{\varepsilon,\left\|\zeta+\left(w_{2}-w_{1}\right) h_{z, w_{1}}(\zeta)-z\right\|\right\}}{r}-\log \frac{\varepsilon}{r} \\
& =\log ^{+} \frac{\left\|\zeta+\left(w_{2}-w_{1}\right) h_{z, w_{1}}(\zeta)-z\right\|}{\varepsilon} \leq \log \left(1+c_{1} \frac{\left\|w_{2}-w_{1}\right\|}{\left\|w_{1}-z\right\|}\right) .
\end{aligned}
$$

Since $g_{D}^{\varepsilon}(z, \cdot)$ is a maximal plurisubharmonic function on $D^{\prime \prime}$ and it is continuous on $\overline{D^{\prime \prime}} \subset \overline{D^{\prime}}$, the domination principle implies that

$$
v(\zeta) \leq c_{2} \frac{\left\|w_{2}-w_{1}\right\|}{\left\|w_{1}-z\right\|}, \quad \zeta \in D^{\prime \prime},
$$

where $c_{2}=\max \left\{c_{1}, c_{3} c_{4}\right\}$. Applying this for $\zeta=w_{1}$ gives (8) .

Proof of Corollary 6. Recall that there is a $c^{\prime}>0$ such that $\tilde{g}_{D}(z, w) \leq c^{\prime}\|z-w\|$, $z \in K, w \in D$. Therefore, we may assume that $w_{1}, w_{2} \neq z$ and $\left\|z-w_{2}\right\| \leq\left\|z-w_{1}\right\|$. Two cases are possible.

Case 1. $\left|g_{D}\left(z, w_{1}\right)-g_{D}\left(z, w_{2}\right)\right|<1$. Then

$$
\begin{aligned}
& \left|\tilde{g}_{D}\left(z, w_{1}\right)-\tilde{g}_{D}\left(z, w_{2}\right)\right|=\tilde{g}_{D}\left(z, w_{2}\right)\left|\exp \left(g_{D}\left(z, w_{1}\right)-g_{D}\left(z, w_{2}\right)\right)-1\right| \\
& \quad<(e-1) c^{\prime}\left\|z-w_{2}\right\| \cdot\left|g_{D}\left(z, w_{1}\right)-g_{D}\left(z, w_{2}\right)\right| \leq(e-1) c^{\prime} C\left\|w_{1}-w_{2}\right\|,
\end{aligned}
$$

where $C$ is the constant from Proposition 5 . 
Case 2. $\left|g_{D}\left(z, w_{1}\right)-g_{D}\left(z, w_{2}\right)\right| \geq 1$. Then, by Proposition [5.

$$
C\left\|w_{1}-w_{2}\right\| \geq\left\|z-w_{2}\right\| \geq\left\|z-w_{1}\right\|-\left\|w_{1}-w_{2}\right\|
$$

and hence $(C+1)\left\|w_{1}-w_{2}\right\| \geq\left\|z-w_{1}\right\|$. It follows that

$$
\begin{aligned}
& \left|\tilde{g}_{D}\left(z, w_{1}\right)-\tilde{g}_{D}\left(z, w_{2}\right)\right|<\max \left\{\tilde{g}_{D}\left(z, w_{1}\right), \tilde{g}_{D}\left(z, w_{2}\right)\right\} \\
& \quad \leq c^{\prime}\left\|z-w_{1}\right\| \leq c^{\prime}(C+1)\left\|w_{1}-w_{2}\right\| .
\end{aligned}
$$

Proof of Proposition 7. By (5), we may find $c>0$ such that if

$$
D_{z, \varepsilon}=\left\{u \in D: \tilde{g}_{D}(z, u)<\varepsilon\right\}, \quad z, \in K, \varepsilon \in(0,1),
$$

then

$$
\operatorname{dist}\left(D_{z, \varepsilon}, \partial D\right) \geq c(1-\varepsilon) .
$$

First, we shall prove (i). By virtue of Corollary [6 it is enough to find a $c_{1}>0$ such that

$$
\left|\tilde{g}_{D}\left(z_{1}, w\right)-\tilde{g}_{D}\left(z_{2}, w\right)\right| \leq c_{1}\left\|z_{1}-z_{2}\right\|, \quad z_{1}, z_{2} \in K, w \in D .
$$

We may assume that $K$ is the closure of a smooth domain. Then there is a $c_{2}>0$ such that for any $z_{1}, z_{2}$ there is a smooth curve $\gamma$ in $K$ joining $z_{1}$ and $z_{2}$ with $l(\gamma) \leq c_{2}\left\|z_{1}-z_{2}\right\|$. Set $\varepsilon=\varepsilon_{z_{1}, z_{2}}=1-\left\|z_{1}-z_{2}\right\| / c$. Then, by integration over $\gamma$, it suffices to prove (10), fixing $w$ and assuming that $1-\left\|z_{1}-z_{2}\right\| / c>\sup _{z \in K} \tilde{g}_{D}(z, w)$. Since $w \in D_{z_{1}, \varepsilon}$ and

$$
D \supset \tilde{D}=\left\{z+z_{2}-z_{1}: z \in D_{z_{1}, \varepsilon}\right\},
$$

then

$$
\tilde{g}_{D}\left(z_{1}, w\right)=\varepsilon \tilde{g}_{D_{z_{1}, \varepsilon}}\left(z_{1}, w\right)=\varepsilon \tilde{g}_{\tilde{D}}\left(z_{2}, w+z_{2}-z_{1}\right) \geq \tilde{g}_{D}\left(z_{2}, w+z_{2}-z_{1}\right)
$$

(cf. [10, Lemma 4.2.7, for the first equality). Hence

$$
\begin{aligned}
& \tilde{g}_{D}\left(z_{2}, w\right)-\tilde{g}_{D}\left(z_{1}, w\right) \leq \tilde{g}_{D}\left(z_{2}, w\right)-\varepsilon \tilde{g}_{D}\left(z_{2}, w+z_{2}-z_{1}\right) \\
& \quad \leq C\left\|z_{2}-z_{1}\right\|+(1-\varepsilon)=(C+1 / c)\left\|z_{2}-z_{1}\right\|,
\end{aligned}
$$

where $C$ is the constant from Corollary 6. By symmetry,

$$
\tilde{g}_{D}\left(z_{1}, w\right)-\tilde{g}_{D}\left(z_{2}, w\right) \leq(C+1 / c)\left\|z_{2}-z_{1}\right\|,
$$

which implies (10).

To prove (ii), it is enough to show that:

- there is a $c_{3}>0$ such that for any $X, Y \in \mathbb{C}^{n}$,

$$
\left|A_{D}(z ; X)-A_{D}(z ; Y)\right| \leq c_{3}\|X-Y\| ;
$$

- if $c_{4}=\max _{z \in K,\|Z\|=1} A_{D}(z ; Z)$, then

$$
\left|A_{D}\left(z_{1} ; X\right)-A_{D}\left(z_{2} ; X\right)\right| \leq c_{4}\|X\| \cdot\|z-w\| / c
$$

for any $z_{1}, z_{2} \in K$ with $\varepsilon=\varepsilon_{z_{1}, z_{2}}>0$ and any $X \in \mathbb{C}^{n}$.

Observe that (11) follows by choosing $c_{3}$ such that

$$
\left|\tilde{g}_{D}\left(z, w_{1}\right)-\tilde{g}_{D}\left(z, w_{2}\right)\right| \leq c_{3}\left\|w_{1}-w_{2}\right\|, \quad z \in K, w_{1}, w_{2} \in D
$$

and using that hyperconvexity implies

$$
A_{D}(z ; X)=\lim _{t \rightarrow 0} \frac{\tilde{g}_{D}(z, z+t X)}{|t|} .
$$


To show (12), we may assume that $A_{D}\left(z_{1} ; X\right) \leq A_{D}\left(z_{2} ; Y\right)$. Since

$$
A_{D}\left(z_{1} ; X\right)=\varepsilon A_{D_{z_{1}, \varepsilon}}\left(z_{1} ; X\right)=\varepsilon A_{\tilde{D}}\left(z_{2} ; X\right) \geq \varepsilon A_{D}\left(z_{2} ; X\right)
$$

(cf. [10], Lemma 4.2.7, for the first equality), then

$$
0 \leq A_{D}\left(z_{2} ; X\right)-A_{D}\left(z_{1} ; X\right) \leq(1-\varepsilon) A_{D}\left(z_{2} ; X\right),
$$

which implies (12).

\section{REFERENCES}

[1] Z. Blocki, The $C^{1,1}$ regularity of the pluriscomplex Green function, Michigan Math. J. 47 (2000), 211-215. MR1793621 (2001k:32057)

[2] Z. Blocki, Regularity of the pluriscomplex Green function with several poles, Indiana Univ. Math. J. 50 (2001), 335-351. MR 1857039 (2002g:32043)

[3] M. Jarnicki, P. Pflug, Invariant distances and metrics in complex analysis, de Gruyter Exp. Math. 9, de Gruyter, Berlin, New York, 1993. MR.1242120 (94k:32039)

[4] M. Jarnicki, P. Pflug, Invariant distances and metrics in complex analysis-revisited, Dissertationes Math. 430 (2005). MR2167637(2006h:32010)

[5] S. G. Krantz, Regularity of the Kobayashi and Carathéodory metrics on Levi pseudoconvex domains, AIM Preprint Series, Volume 10 (2007).

[6] S. G. Krantz, Pseudoconvexity, analytic discs, and invariant metrics, AIM Preprint Series, Volume 10 (2007).

[7] N. Nikolov, P. Pflug, Local vs. global hyperconvexity, tautness or k-completeness for unbounded open sets in $\mathbb{C}^{n}$, Ann. Scuola Norm. Sup. Pisa Cl. Sci. (5), IV (2005), 601-618. MR2207736 (2006j:32002)

[8] N. Nikolov, P. Pflug, On the definition of the Kobayashi-Buseman pseudometric, Internat. J. Math. 17 (2006), 1145-1149. MR2287671 (2007i:32011)

[9] N. Nikolov, P. Pflug, On the derivatives of the Lempert functions, Ann. Mat. Pura Appl. (4) 187 (2008), 547-553. MR2393147

[10] W. Zwonek, Completeness, Reinhardt domains and the method of complex geodesics in the theory of invariant functions, Dissertationes Math. 388 (2000). MR 1785672 (2001h:32016)

Institute of Mathematics and Informatics, Bulgarian Academy of Sciences, Acad. G. Bonchev 8, 1113 Sofia, Bulgaria

E-mail address: nik@math.bas.bg

Carl von Ossietzky Universität Oldenburg, Institut für Mathematik, Postfach 2503, D-26111 Oldenburg, Germany

E-mail address: pflug@mathematik.uni-oldenburg.de

Institut de Mathématiques, Université Paul Sabatier, 118 Route de Narbonne, 31062 Toulouse Cedex 9, France

E-mail address: pthomas@cict.fr 